\title{
Theoretical strength of 2D hexagonal crystals: application to bubble raft indentation
}

\author{
S. V. DMITRIEV*†+, J. LI§, N. YOSHIKAWA $\dagger$ \\ and Y. SHIBUTANI \\ $\dagger$ Institute of Industrial Science, The University of Tokyo, 153-8505 Tokyo, Japan \\ \$National Institute of Materials Science, 1-2-1 Sengen, Tsukuba, \\ Ibaraki 305-0047, Japan \\ $\S$ Department of Materials Science and Engineering, Ohio State University, \\ Columbus, OH 43210, USA \\ \Department of Mechanical Engineering and Systems Graduate School of \\ Engineering, Osaka University, 565-0871 Osaka, Japan
}

(Received 2 April 2004; in final form 27 October 2004)

\begin{abstract}
By means of lattice and molecular dynamics we study the theoretical strength of homogeneously strained, defect-free $2 \mathrm{D}$ crystals whose atoms interact via pair potentials with short- and longer-ranged interactions, respectively. We calculate the instability surface, i.e. the boundary in the 3D homogeneous strain space $\left(\varepsilon_{x x}, \varepsilon_{y y}, \varepsilon_{x y}\right)$, at which the first vanishing of the frequency of a vibrational mode occurs, taking into account all $2(N-1)+3$ modes of a $2 \mathrm{D}$ periodic system of $N$ atoms. We also compute the strain energies of the crystal on the instability surface, thus defining the most dangerous direction(s) of strain where the critical energy density is small. A theory is developed to incorporate the effect of loading device-sample interactions in the lattice instability criterion. The results are applied to the model problem of bubble raft indentation. We analyse the distribution of the unstable phonon modes in the first Brillouin zone as a function of the loading parameter, and discuss the post-critical behaviour of the lattice in the presence of strain gradients as in nanoindentation experiments.
\end{abstract}

\section{Introduction}

In theoretical strength studies, one determines the domain of homogeneous strain and temperature in which a perfect crystal is stable. This problem has been extensively studied in physics in relation to the pressure- and/or temperature-induced structural phase transformation in solids $[1,2]$ and also in elasticity theory and structural mechanics in the context of elastic body instability [3, 4]. The huge gap between the theoretical strength estimated for defect-free crystals and the measured yield stresses of real materials stimulated the development of dislocation theory.

*Corresponding author. Email: sergey@iis.u-tokyo.ac.jp 
The Peierls-Nabarro model of the dislocation core [5, 6] based on earlier work of Frenkel [7] reconciles the above phenomena and reveals deep connections between ideal strength and defect mobility. Defects like the dislocation core and crack tip provide the necessary leverage to amplify an external shear stress to the level that can break bonds locally, albeit in an inhomogeneous and asynchronous fashion. Thus, the study of theoretical strength is an important area at the crossroad of several fields. The first practical application of theoretical strength was related to whiskers, nominally dislocation-free filamentary crystals [8]. Nano-indentation experiments [9-11] have awakened fresh interest in the theoretical strength problem [12, 13]. In such experiments on nearly perfect crystals the measured load-displacement response shows characteristic discontinuities attributed to the discrete generation of crystal defects, which can be likened to observations of 'quanta' of plastic deformation. Surprisingly, defects can be generated not only at the contact surface, but also in the bulk of a defect-free single crystal.

Theoretical strength can be discussed in the framework of a microscopic analysis or a phenomenological continuum theory. The former approach is exact while the latter depends on various assumptions to coarse-grain the discrete system to a continuum. Microscopic analysis requires the knowledge of interaction forces between atoms, and realistic interatomic potentials are now available for a few materials. An advantage of the phenomenological theory is that it deals with the physical characteristics measurable in a macroscopic experiment such as elastic moduli, strain, stress, and temperature.

While the analysis of stability of an equilibrium state is a linear problem, the nonlinear theory of finite strain [3] is needed to calculate the pre-critical behaviour of the system. In principle, one can avoid the use of the finite strain elasticity by using the incremental elasticity [14]. In this approach, the nonlinear pre-critical behaviour is calculated as a sequence of linear problems for sufficiently small increments of the external load with the correction in the geometry of the system and in the accumulated initial stress at each step.

Let us turn to the discussion of the criteria of instability, but first we need to define the concept of 'stability' or 'instability'. Here we are interested in the stability of an equilibrium state (in some other fields the instability of motion can be of interest), which is called undisturbed equilibrium. In addition, we will consider a disturbed form of motion or, in the case of potential external load, it is sufficient to consider a disturbed form of equilibrium [3]. An equilibrium state is defined to be stable with respect to a small disturbance if, for smaller amplitude of disturbance, the deviation from the undisturbed state does not grow with time.

In the theoretical strength problem we are dealing with an infinite crystal under slowly changing homogeneous strain and/or temperature. In this context the problem has been extensively studied in the theory of structural phase transitions in solids and there exists an enormous literature on this subject $[1,2,15-19]$. The lattice instability theory currently developed can accept many results of the theory of structural phase transitions. The relationship between structural phase transitions and the dynamical properties of lattices in the higher symmetry phase was first pointed out explicitly by Anderson [15] and by Cochran [16]. From that time on the soft mode concept has served as a criterion of instability (phase transition) in a phenomenological treatment.

The harmonic phonon system does not change its eigen-frequencies upon a change of temperature to become soft and, thus, the linear phonons themselves 
cannot cause a phase transition. From the microscopic point of view, Yamada [17] categorized the phase transitions into two classes. The first class of lattice instability is due to the anharmonic coupling of phonons which can take place at a high temperature when the amplitudes of the atomic vibrations are not small. The second class is the lattice instability in coupled systems. Here the harmonic phonons are coupled strongly to other physical variables, e.g. lattice strain. In the first class of instability the temperature plays a dominant role, while in the second type the finite temperature may only lower the critical loads, but the essential features of the instability can be understood even at zero temperature. In this paper we will consider the second type of lattice instability and neglect the temperature effect, which is acceptable especially for the bubble raft where any dynamics is damped.

It is, of course, desirable to express the lattice instability criterion in terms of continuum mechanics and it has been done, first for the instability with respect to homogeneous strains, B-criterion [20], and later for the instability with respect to a long-wave phonon mode, $\Lambda$-criterion [13]. Some difficulties of the continuum consideration have been pointed out, e.g. Hill and Milstein [21] noted the dependence of the instability criterion on the parameters used to describe the crystal strain, and Wang et al. [20] observed the path dependence of the instability criterion for the so-called 'constant-stress' ensemble. Microscopically, for interatomic potentials depending only on the coordinates of the atoms, there is no path dependence of the internal energy of the crystal. In quasi-equilibrium loading, internal energy is equal to work done by the external loads and, consequently, the criterion of instability of an equilibrium state cannot be path-dependent, although it may depend on the behaviour of the external load in response to a small deviation from the undisturbed equilibrium.

In the present paper the lattice instability will be treated purely microscopically, at the atomic level. In this case the application of the soft mode criterion implies the calculation of the frequencies of all $2(N-1)+3$ linear vibration modes for a $2 \mathrm{D}$ crystal having $N$ atoms $(3(N-1)+6$ modes for a $3 \mathrm{D}$ crystal $)$. It is convenient to treat separately the three modes corresponding to homogeneous strains from the $2(N-1)$ phonon modes. The loss of stability with respect to a homogeneous strain mode requires taking into account the work of external loads done on the corresponding 'macroscopic' displacements. In contrast, the small amplitude vibration modes do not cause thermal expansion or other changes in geometry and the external loads do not produce work. Instability with respect to a homogeneous strain mode can be called 'bulk' instability, and the corresponding criterion of instability is conveniently called the B-criterion with the mnemonic B standing for bulk. We treat the bulk instability microscopically, but the B-criterion was first presented phenomenologically [20]. Similarly, the criterion which checks for instability with respect to a phonon mode is conveniently called the P-criterion. The P-criterion is similar to the $\Lambda$-criterion introduced by Van Vliet [13] phenomenologically to check for instability with respect to a long-wave phonon mode (vanishing of sound velocity). The P-criterion, which is just the phonon soft mode criterion, is applied not only to long- but also to short-wave phonons and it has a microscopic origin, i.e. when formulating the corresponding eigen-value problem, there is no need to introduce the notion of strain, stress, and elastic moduli.

It is also important to note that the fact of the instability of a crystal determined from the linearized equations does not necessarily mean structural collapse. It is well known that many soft-mode-driven structural phase transitions in crystals with 
more or less complex structures have only small effects on the macroscopic physical properties. In the spirit of the theoretical strength investigations such critical points should be ignored. Thus, analysis of the post-critical behaviour is the problem of crucial importance. However, this problem is not that important for closedpacked metals and also for the closed-packed 2D lattices studied here, because they have primitive structures.

In the case of nano-indentation we have to take into account the boundary conditions, since the instability takes place in a local volume in the presence of the strain gradients. This is another peculiarity of the lattice instability problem compared with the theory of temperature-driven or larger length-scale phase transitions.

In the present paper we discuss the instability of a two-dimensional defectfree homogeneously strained lattice with atoms interacting via pair potentials of two types, the Lennard-Jones potential and a short-range potential qualitatively describing the interaction of bubbles in the bubble raft model of the crystal [12]. We subject the lattice to a homogeneous strain $\left(\varepsilon_{x x}, \varepsilon_{y y}, \varepsilon_{x y}\right)=\alpha\left(\varepsilon_{x x}^{0}, \varepsilon_{y y}^{0}, \varepsilon_{x y}^{0}\right)$, where the vector $\left(\varepsilon_{x x}^{0}, \varepsilon_{y y}^{0}, \varepsilon_{x y}^{0}\right)$ has unit norm and $\alpha>0$. We systematically study the dispersion relations for the small-amplitude oscillation modes and the three frequencies corresponding to the homogeneous strain modes, thus defining the instability surface in the three-dimensional strain space, the surface at which the first imaginary frequency appears. Then we calculate the cohesive energy at the points of the critical surface and find the most dangerous combinations of the homogeneous strain components. This should be the direction of strain that the material is most vulnerable to. Thus we expect, for brittle materials, the most vulnerable strain direction is of tensile nature, while, for ductile materials, it is of shear nature. The approach used here is purely microscopic and the results obtained do not depend on a particular definition of finite strain or a definition of stress at finite strain.

\section{Simulation details}

\subsection{Geometry of the system}

The two-dimensional hexagonal lattice with lattice parameter $a_{0}$ is generated by the vectors $\mathbf{p}_{0}=a_{0}(1,0)$ and $\mathbf{q}_{0}=a_{0}(1 / 2, \sqrt{3} / 2)$.

\subsection{Application of homogeneous strain}

We subject the lattice to the homogeneous strain with components $\left(\varepsilon_{x x}, \varepsilon_{y y}, \varepsilon_{x y}\right)=$ $\alpha\left(\varepsilon_{x x}^{0}, \varepsilon_{y y}^{0}, \varepsilon_{x y}^{0}\right)$, where the vector $\left(\varepsilon_{x x}^{0}, \varepsilon_{y y}^{0}, \varepsilon_{x y}^{0}\right)$ has unit length and $\alpha>0$. The generator vector of the strained lattice is $\mathbf{p}=\mathbf{p}_{0}+\mathbf{p}_{0} \mathbf{H}, \mathbf{q}=\mathbf{q}_{0}+\mathbf{q}_{0} \mathbf{H}$, where matrix $\mathbf{H}$ has coefficients $h_{11}=\alpha \varepsilon_{x x}^{0}, h_{12}=\alpha \varepsilon_{x y}^{0} / 2, h_{21}=\alpha \varepsilon_{x y}^{0} / 2, h_{22}=\alpha \varepsilon_{y y}^{0}$, so that the $(i, j)$ th atom has the position vector $\mathbf{r}_{i j}=i \mathbf{p}+j \mathbf{q}$. One can see that here we use the linear strain tensor, $\varepsilon_{i j}$. This is sufficient for our purpose, because, in the formulation of the instability eigen-value problem, we actually use the atomic coordinates but not the strain tensor and the latter is used only as a convenient way of parameterization. Due to the rotational invariance, only three of the four components of the vectors $\mathbf{p}$ and $\mathbf{q}$ are independent, so that any deformed state can be represented by a point in three-dimensional space. We can choose different coordinates, for example lengths of the vectors $\mathbf{p}$ and $\mathbf{q}$, and the angle between them, but we prefer to use the three 
components of the symmetric strain tensor, $\varepsilon_{x x}, \varepsilon_{y y} \varepsilon_{x y}$, to describe the geometry of the homogeneously deformed crystal. Our results obtained in the space of components of the linear strain tensor can be readily transformed to any other measure of strain.

\subsection{Interatomic interactions}

For the sake of comparison we take two different pair potentials, a long-range and a short-range potential with only nearest-neighbour interactions. We would like to check if the use of these two potentials would give some qualitative changes in the mechanisms of lattice instability.

First is the Lennard-Jones (LJ) pair potential,

$$
\varphi(r)=4 \varepsilon\left[\left(\frac{\sigma}{r}\right)^{12}-\left(\frac{\sigma}{r}\right)^{6}\right],
$$

where $r$ is the distance between two atoms and, without loss of generality, we set for the parameters $\varepsilon=1 / 4$ energy units and $\sigma=1$ length units. We also normalize the mass of an atom to unity, which can always be done by proper choice of the time unit. For a cut-off radius equal to 11, the equilibrium lattice parameter is $a_{0}=1.11146206$. The cohesive energy per atom in the unstrained crystal is $E_{0}=-0.845459$. In the following we always calculate energies per atom. The potential energy of the crystal is sometimes defined as $P=E-E_{0}$ and sometimes as $P=E-E^{*}$, where $E$ is the cohesive energy of the crystal, $E_{0}$ is the cohesive energy of the unstrained crystal given above, and $E^{*}$ is the cohesive energy of the homogeneously strained crystal at $r=r^{*}$, i.e. at the magnitude of strain parameter $\alpha$ where the first imaginary frequencies in the vibration spectrum appear.

Another potential is similar to that used by Van Vliet [13] to simulate bubble raft indentation. We modify that potential to eliminate the discontinuity in $\varphi^{\prime \prime}(r)$, which is important in the theory of instability, and assume for the bubble raft (BR) potential

$$
\varphi(r)= \begin{cases}\frac{\left(r-r_{\mathrm{c}}\right)^{8}}{\left(r_{\mathrm{b}}-r_{\mathrm{c}}\right)^{8}}-\frac{2\left(r-r_{\mathrm{c}}\right)^{4}}{\left(r_{\mathrm{b}}-r_{\mathrm{c}}\right)^{4}}, & r<r_{\mathrm{c}}, \\ 0, & r \geq r_{\mathrm{c}},\end{cases}
$$

where we have chosen the unit of length to be equal to the bubble radius, $r_{\mathrm{b}}=1$, and for the cut-off radius we set $r_{\mathrm{c}}=1.3$. The energy unit is chosen in such a way that $\varphi\left(r_{\mathrm{b}}\right)=-1$. The BR potential, in contrast to the LJ potential, is a short-ranged potential, each bubble interacting only with the nearest neighbours. The cohesive energy of the unstrained bubble raft is equal to $E_{0}=-3$ per bubble.

\subsection{Indentation simulations}

We consider two films of different crystal orientations, referred to as systems 1 and 2 , respectively. System 1 (2) contains $N=2880$ (2856) atoms, $L_{x}=60 a_{0}=66.69$ $\left(3^{1 / 2} 34 a_{0}=65.45\right)$ and $L_{y}=3^{1 / 2} 24 a_{0}=46.20\left(42 a_{0}=46.68\right)$. The top face of the film is a free surface, the bottom face interacts with the rigid continuation of the crystal, and we employ the periodic boundary conditions in the horizontal direction. Systems 1 and 2 are indented along directions $\langle 112\rangle$ and $\langle 110\rangle$, respectively. In this study we use a rigid half-sphere tip with diameter $56 a_{0}$. There is no friction between the 
indenter and the material. We introduce a viscosity term into the equations of motion of the atoms in order to study their relaxation to energy minimum positions. As such, the temperature effect is not taken into account.

\section{Stability of the homogeneously strained lattice}

\subsection{Critical surfaces in the strain space}

The linear vibration spectrum of a 2D crystal having $N$ atoms consists of $2(N-1)$ phonon modes plus three homogeneous strain modes. Application of the P-criterion of lattice instability implies the calculation of the frequencies of $2(N-1)$ smallamplitude phonon modes, $\mathbf{U}(t)=\mathbf{U}^{0} \exp \left[\mathrm{i}\left(k_{x} l+k_{y} m-\omega t\right)\right]$, where $\mathrm{i}$ is the imaginary unit, $0 \leq k_{x}, k_{y} \leq 2 \pi$ are the components of a wavevector in the first Brillouin zone, $l, m$ are integer numbers specifying a periodic cell, $\omega$ is the frequency of a particular phonon mode, and $\mathbf{U}^{0}$ is the corresponding time-independent eigen-vector containing two components of the displacement vector of the atom in the periodic cell. Stability with respect to the P-criterion is lost when a phonon with imaginary frequency appears in the spectrum, which means a change from oscillatory motion near the stable equilibrium to an exponential (in time) deviation from the unstable equilibrium. To find the unstable phonons we scan the first Brillouin zone with the steps $\Delta k_{x}=\Delta k_{y}=0.01 \pi$.

The B-criterion is used to check the stability of the crystal lattice with respect to a homogeneous strain mode (three remaining degrees of freedom). The eigen-value problem here is formulated with respect to three parameters specifying the shape and size of the primitive translational cell.

Thus, we calculate the phonon spectrum and the frequencies of the three homogeneous strain eigen-modes for the 2D crystal strained with the components of the strain tensor, $\alpha\left(\varepsilon_{x x}^{0}, \varepsilon_{y y}^{0}, \varepsilon_{x y}^{0}\right)$, where the unit length vector $\left(\varepsilon_{x x}^{0}, \varepsilon_{y y}^{0}, \varepsilon_{x y}^{0}\right)$ defines a direction in the three-dimensional strain space and $\alpha>0$ is a parameter defining the strength of the deformation. For different strain relations, $\left(\varepsilon_{x x}^{0}, \varepsilon_{y y}^{0}, \varepsilon_{x y}^{0}\right)$, we numerically define the critical value, $\alpha^{*}$, at which the first eigen-frequency of the vibration spectrum (P-criterion) or a homogeneous strain mode (B-criterion) vanishes. The corresponding eigen-mode gives the instability mode of the crystal. We also calculate the critical increase of the cohesive energy per atom, $E^{*}-E_{0}$, at point $\alpha^{*}$.

When using the B-criterion, we assume that the external loads do not change in magnitude or direction when the system deviates slightly from the undisturbed equilibrium.

Critical values $\alpha^{*}$ and $E^{*}-E_{0}$ make surfaces in the three-dimensional strain space. In figures 1 and 2 we give the three sections of these surfaces, $\varepsilon_{x x}=0, \varepsilon_{y y}=0$, and $\varepsilon_{x y}=0$, for the BR and LJ potentials, respectively. The left columns of figures 1 and 2 show $\alpha^{*}$ and the right columns show $E^{*}-E_{0}$. The P-criterion is fulfilled at the solid lines and the B-criterion at the dashed lines.

The critical surfaces separate the stable and unstable regions in the threedimensional strain space. The distance from the origin, which corresponds to the unstrained crystal, to a point of a critical surface specifies the magnitude of the critical parameter, where the relation between strain components is proportional to the relation between coordinates of the point. 

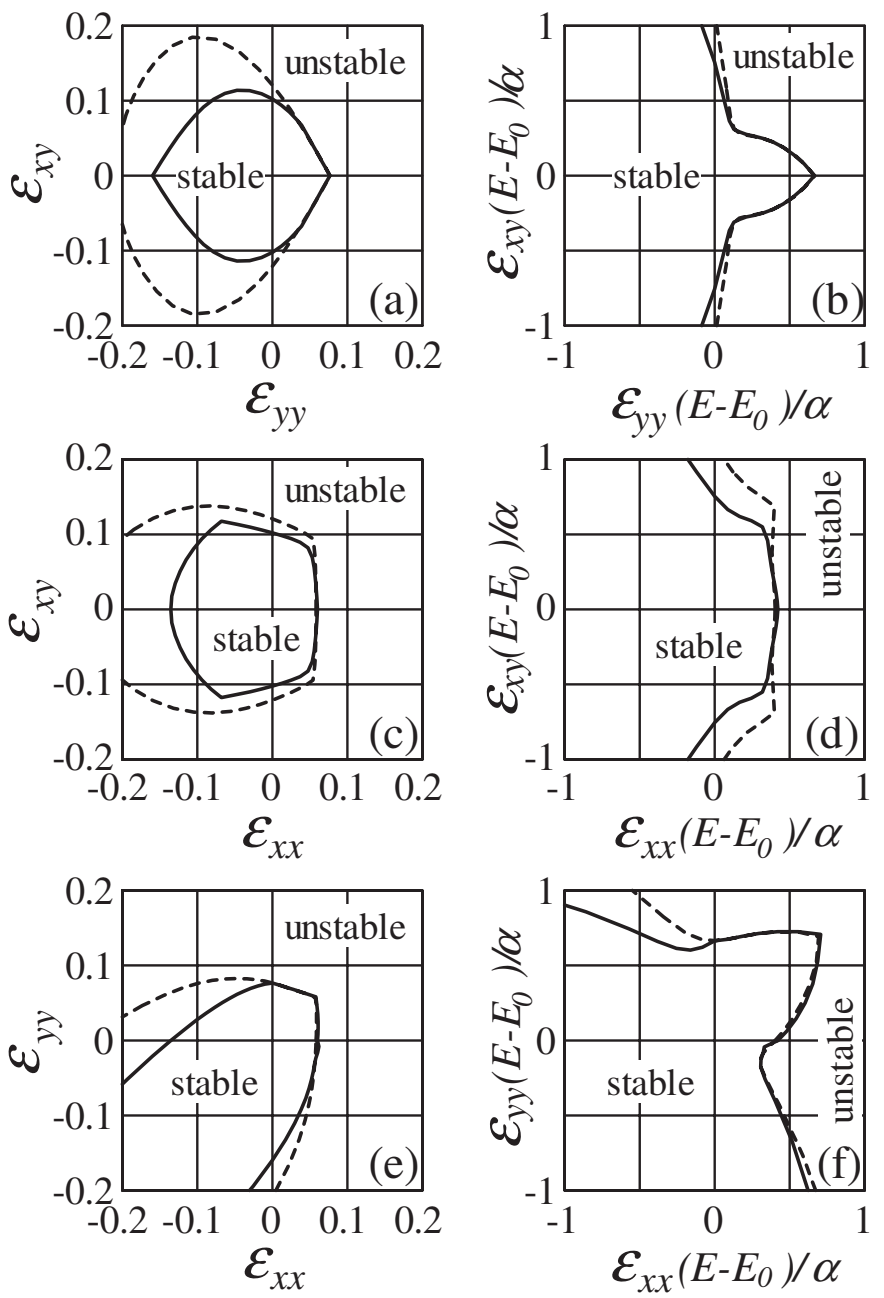

Figure 1. Critical surfaces in the three-dimensional strain space represented by the three sections corresponding to $(\mathrm{a}, \mathrm{b}) \varepsilon_{x x}=0$, (c, d) $\varepsilon_{y y}=0$ and (e, f) $\varepsilon_{x y}=0$. The distance from the origin to a point of the instability boundary in the left column shows $\alpha^{*}$ and in the right column $E^{*}-E_{0}$. Instability with respect to a linear phonon mode (P-criterion) takes place at the solid lines and with respect to a homogeneous strain mode (B-criterion) at the dashed lines. The results are for the BR potential.

The following features of the critical surfaces should be noted.

(i) The quasi-static deformation of a crystal with potential energy depending only on the coordinates of the atoms is path-independent and, in this sense, the critical surfaces presented in figures 1 and 2 are the universal characteristics of the theoretical strength.

(ii) The critical surfaces for the BR potential (figure 1) and the LJ potential (figure 2) have the same topology in spite of the considerable difference in the interatomic potentials. This is a reflection of the fact that both $2 \mathrm{D}$ crystals have the same symmetry. It would be instructive to construct 

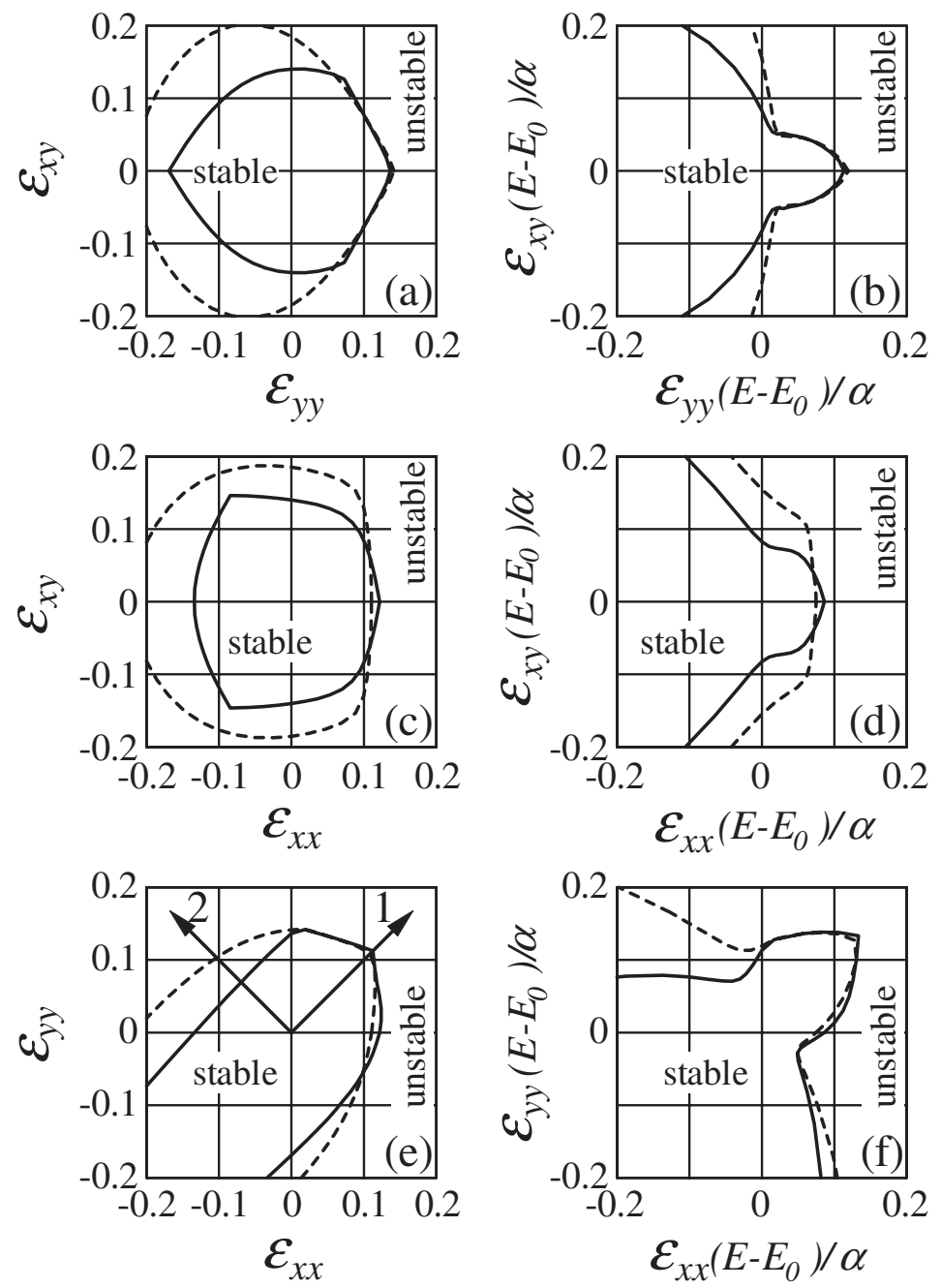

Figure 2. Same as figure 1, but for the Lennard-Jones potential.

similar surfaces for representative materials, for example for typical fcc, bcc, and hcp metals and other materials.

(iii) The critical strain energy surface, $E^{*}-E_{0}$, is very important in theoretical strength studies and in the nano-indentation problem because it predicts the strain conditions at which lattice instability is most readily inducible. The points that are closest to the origin in figures $1 \mathrm{~b}, \mathrm{~d}$ and $\mathrm{f}$ and figures $2 \mathrm{~b}, \mathrm{~d}$ and $\mathrm{f}$ are the most dangerous strain directions because, here, comparatively small energy is required to destroy the crystal. For the BR potential, the smallest critical energy (distance from the origin) is $E^{*}-E_{0}=0.33$ (see figure $1 \mathrm{~b}$ ), which corresponds to $\alpha^{*}\left(\varepsilon_{x x}^{0}, \varepsilon_{y y}^{0}, \varepsilon_{x y}^{0}\right)=$ $0.079(0,1 / \sqrt{3}, 2 / \sqrt{3})$. For the LJ crystal these values are $E^{*}-E_{0}=0.055$ and $\quad \alpha^{*}\left(\varepsilon_{x x}^{0}, \varepsilon_{y y}^{0}, \varepsilon_{x y}^{0}\right)=0.143(0,4 / \sqrt{116}, 10 / \sqrt{116})$ (see figure $2 \mathrm{~b}$ ). Physically, for both potentials in the $2 \mathrm{D}$ closed-packed lattice, the most dangerous strain direction is shear along the closed-packed atomic chains 
in combination with a small tension perpendicular to these chains. This is in good agreement with the actual unstable phonon eigen-vector found in 2D bubble-raft indentation simulations $[13,22]$ under strong compressive loading conditions, in which it was found that there is approximately $12^{\circ}$ between the phonon polarization vector $\mathbf{w}$ and the Burgers vector $\mathbf{b}$, indicating shear-tension coupling at the shear-dominated elastic instability. The second most dangerous type of loading for both crystals is the uniaxial tensile strain $\varepsilon_{x x}$ (along the closed-packed atomic chains). In this case, $E^{*}-E_{0}=0.40, \alpha^{*}=0.058$ for the BR potential (see figure 1d), and $E^{*}-E_{0}=0.075, \alpha^{*}=0.11$ for the LJ potential (see figure $2 \mathrm{~d}$ ). This mode corresponds to cleavage failure. Because the energy cost is $20-35 \%$ higher than that of the shear failure mode, and since indentation creates a predominantly compressive stress condition, we do not observe brittle crack nucleation in our indentation simulations [13, 22] nearly as often as dislocation nucleation. Interestingly, by changing the only dimensionless parameter $r_{0}$ of the original BR potential [13] from 0.85 to higher values, we have observed increasing brittle activity. This phenomenon is associated with the approach or even crossing-over of $E^{*}-E_{0}$ of the second (tensile) mode with that of the first (shear) mode.

(iv) For some combinations of homogeneous strain, the P-criterion and the B-criterion give considerably different critical values. However, it is remarkable that, for the most dangerous cases described in (iii), both the $\mathrm{P}$-criterion and the B-criterion give very similar critical values $\alpha^{*}$ and $E^{*}-E_{0}$.

(v) A change in the symmetry of the crystal during the pre-critical deformation is very important. An unstrained 2D hexagonal crystal is isotropic, but, for example, the uniaxial strains $\varepsilon_{x x}$ (along the closed-packed atomic chains) and $\varepsilon_{y y}$ (perpendicular to the closed-packed atomic chains) show a noticeable difference in critical parameters (see figures $1 \mathrm{e}$ and $\mathrm{f}$ and figures $2 \mathrm{e}$ and $\mathrm{f}$ ).

(vi) As seen from figures $1 \mathrm{e}$ and $2 \mathrm{e}$, the critical surfaces are unbounded. This is because no lattice instability is observed in the hydrostatic compression condition, $\left(\varepsilon_{x x}^{0}, \varepsilon_{y y}^{0}, \varepsilon_{x y}^{0}\right)=(-1 / \sqrt{2},-1 / \sqrt{2}, 0)$, of the closed-packed 2D crystal. In reality, of course, there are many pressure-induced phase transitions for materials with complex structures.

(vii) From the analysis of the eigen-modes we found that, in all cases, instability with respect to a linear phonon mode (P-criterion) indicates the vanishing of sound velocity in a particular direction or, in other words, an acoustic branch always vanishes at the $\Gamma$-point, i.e. at the origin of the first Brillouin zone, $\mathbf{k}=0$. We obtained the whole critical surface, not only the three sections presented in figures 1 and 2 , and instability with respect to a mode with $\mathbf{k} \neq 0$ was not observed in any of the $\left(\varepsilon_{x x}^{0}, \varepsilon_{y y}^{0}, \varepsilon_{x y}^{0}\right)$ combinations. This is consistent with the general trend that the incommensurate phase can be more easily realized in crystals with more or less complex structure [18], especially for crystals consisting of comparatively rigid atomic groups with rotational degrees of freedom [23-25], although there can be exceptions [26]. Theoretically, incommensurate modulation can appear in a simple lattice [27], but, to the best of our knowledge, no incommensurate phase has been discovered in a pure metal. 


\subsection{Sample-loading device interaction}

It is well known that the behaviour of the loading device when the body is perturbed slightly from its equilibrium position can have a significant effect on its stability (or instability) with respect to a homogeneous strain mode (B-criterion). This problem has been addressed as the problem of the interaction of the sample with the loading device $[3,4,20,21,28]$. If the external forces have a potential (conservative forces), then, at the point of instability, purely imaginary frequencies will appear in the linear excitation spectrum and the disturbance will then grow monotonically with time (static instability). This may also occur for non-conservative forces, but, in this case, there is another possibility, namely one can observe an oscillatory instability when, at a critical point, two real frequencies merge and then transform into a complex conjugate pair and the increase in the disturbance is of an oscillatory nature [3].

When the loading device can be regarded as an elastic body with fixed boundary conditions, the external load has a potential or, in other words, it is conservative. This is particularly important for the analysis of nano-indentation experiments, since the local area of the lattice where the instability occurs interacts with the surrounding stable medium, which behaves elastically.

Let us now take into account the interaction of the loading device with the sample. We consider a perfect crystal deformed with homogeneous strain $\varepsilon_{i j}$, and the stress components at this state are $\sigma_{i j}$. To apply the B-criterion of instability we consider an infinitesimally close strain state, $\varepsilon_{i j}+\delta \varepsilon_{i j}$. We write

$$
\delta E=\delta A,
$$

where $\delta E$ is the change in elastic energy density of the crystal due to the strain increment $\delta \varepsilon_{i j}$, and $\delta A$ is the work done by the unit volume of the crystal against the external load. The magnitude of $\delta A$ depends on how the crystal interacts with the loading device. For example, let us imagine an experiment on the structural transformation in a crystal under hydrostatic pressure. The sample is placed in a chamber containing an absolutely incompressible liquid. We increase the pressure $p$ in the chamber by injecting very small portions of liquid and inspect the system after adding each new portion. We consider a transition accompanied by volumetric deformation $\delta V$. When the crystal volume starts to decrease at a critical point, the energy stored by a very elastic chamber will keep the pressure almost constant and $\delta A=p \delta V$. The more rigid the chamber, the faster the pressure decreases due to the change in crystal volume. Assuming that the pressure decreases linearly with $\delta V$, one has $\delta A=\mathrm{p} \delta V-(\gamma / 2)(\delta V)^{2}$, where $\gamma>0$ is the coefficient describing the rigidity of the chamber.

Our problem is to find the conditions for which the potential energy of the system is stationary for a non-trivial $\delta \varepsilon_{i j}$. For a two-dimensional crystal, the three stationary conditions $\delta(\delta E-\delta A) / \delta\left(\delta \varepsilon_{x x}\right)=0, \delta(\delta E-\delta A) / \delta\left(\delta \varepsilon_{y y}\right)=0$ and $\delta(\delta E-\delta A) / \delta\left(\delta \varepsilon_{x y}\right)=0$ lead to the expressions

$$
\begin{aligned}
& \varepsilon_{x x}\left(C_{11}-\sigma_{x x}+\gamma_{1}\right)+\varepsilon_{y y} C_{12}+\varepsilon_{x y}\left(C_{13}-\frac{1}{2} \sigma_{x y}\right)=0, \\
& \varepsilon_{x x} C_{12}+\varepsilon_{y y}\left(C_{22}-\sigma_{y y}+\gamma_{2}\right)+\varepsilon_{x y}\left(C_{23}-\frac{1}{2} \sigma_{x y}\right)=0, \\
& \varepsilon_{x x}\left(C_{13}-\frac{1}{2} \sigma_{x y}\right)+\varepsilon_{y y}\left(C_{23}-\frac{1}{2} \sigma_{x y}\right)+\varepsilon_{x y}\left(C_{33}-\frac{1}{4}\left(\sigma_{x x}+\sigma_{y y}\right)+\gamma_{3}\right)=0,
\end{aligned}
$$


where $\gamma_{1}, \gamma_{2}, \gamma_{3}$ describe the rigidity of the loading device with respect to the stress components $\sigma_{x x}, \sigma_{y y}, \sigma_{x y}$, respectively, and $C_{i j}$ are the elastic moduli in the current state.

Equations (4) can have a non-trivial solution only if the determinant of the system is zero. Applying this to the hydrostatic tension, $\sigma_{x x}=\sigma_{y y}=p>0, \sigma_{x y}=0$, of an isotropic crystal, $C_{11}=C_{22}, C_{33}=\left(C_{11}=C_{22}\right) / 2, C_{13}=C_{23}=0, C_{i j}=C_{j i}$, we come to the stability conditions

$$
\begin{aligned}
& C_{11}+C_{12}-p+\gamma>0, \\
& 2 C_{33}-p>0,
\end{aligned}
$$

where, for hydrostatic loading, we have set $\gamma_{1}=\gamma_{2}=\gamma, \gamma_{3}=0$.

Violation of the first condition in (5) results in spinodal decohesion and, unlike the second criterion for shear instability, it depends on the rigidity of the chamber, $\gamma$. Obviously, for a chamber of finite rigidity $(\gamma>0)$, the crystal is more stable with respect to spinodal decohesion than for an absolutely elastic chamber $(\gamma=0)$.

To verify our conclusions we consider the hydrostatic tension of a $2 \mathrm{D}$ crystal with the LJ interatomic potential. At zero temperature we find that, for an absolutely elastic chamber $(\gamma=0)$, the B-criterion (first condition of (5)) gives the critical lattice parameter $a_{\mathrm{B}}=1.2323$. The second condition of (5) is not important because it is satisfied at a considerably larger lattice parameter, 1.2476. The P-criterion suggests an instability (vanishing of sound velocity) at $a_{\mathrm{P}}=1.2361$. Thus, under this loading condition, one would expect spinodal decomposition at $a=a_{\mathrm{B}}$. However, at a finite rigidity of the chamber the critical value will be greater than $a_{\mathrm{B}}$. Already at $\gamma=0.2629$, one has $a_{\mathrm{B}}=a_{\mathrm{P}}$, and for a larger $\gamma$, violation of the P-criterion would be responsible for the instability.

After the above consideration, we note that the results presented in figures 1 and 2 were obtained under the assumption that the external loads do not change during deviation from the undisturbed equilibrium, which corresponds to an absolutely elastic loading device, $\gamma=0$. However, in nano-indentation experiments, in the presence of strain gradients, instability occurs in a local area. This area interacts with the surrounding stable material, which can be regarded as a loading device with finite rigidity, $\gamma>0$. In this case, violation of the B-criterion will be delayed and the P-criterion may become responsible for the instability. This is particularly important for a 2D hexagonal lattice since, for this case, we found a rather small difference in the critical values obtained from the P-criterion and the B-criterion at $\gamma=0$ (see item (iv) in section 3.1). In the analysis of the nanoindentation results presented in section 4 , we only take into account the P-criterion.

\subsection{Post-critical behaviour}

Mode softening does not necessarily mean collapse of the crystal lattice. The critical point is determined from the linearized equations, but the post-critical behaviour is controlled by the full crystal potential. In nano-indentation experiments, there exists a gradient of strains and the instability has local character so that boundary conditions should be taken into account. Here we study the post-critical behaviour for the hydrostatic tension and for the pure shear strain taking into account the size effect. We carry out the MD simulation for a computational cell containing $N \times N$ atoms and subjected to periodic boundary conditions. A small temperature, $T=0.00018$, for hydrostatic tension and $T=0.005$ for pure shear were introduced to check 
the stability. Temperature is defined as twice the kinetic energy density of the crystal. At these temperatures the amplitude of the atomic displacements is about $0.01 a_{0}$. We control strain, but not the external stress as in the Parinello-Rahman scheme. This corresponds to an infinitely rigid loading device.

We found that the LJ and BR interatomic potentials give qualitatively similar results and, in this section, we present the results for the LJ potential only.

As an example, the kinetics of the post-critical behaviour of the LJ crystal is presented in figure 3 for a computational cell with $N=16$ and for hydrostatic tension $\alpha\left(\varepsilon_{x x}^{0}, \varepsilon_{y y}^{0}, \varepsilon_{x y}^{0}\right)=\alpha(1 / \sqrt{2}, 1 / \sqrt{2}, 0)$, at $\alpha=\alpha_{\mathrm{HT}}^{*}-0.0022$, where $\alpha_{\mathrm{HT}}^{*}=0.1597$ is the critical value for the P-criterion. Note that the B-criterion at $\gamma=0$ gives a smaller critical value, 0.1538 , but here we will use the P-critical values for both cases, pure shear and hydrostatic tension. Figure 4 presents the same as in figure 3, but for pure shear strain, $\alpha\left(\varepsilon_{x x}^{0}, \varepsilon_{y y}^{0}, \varepsilon_{x y}^{0}\right)=\alpha(-1 / \sqrt{2}, 1 / \sqrt{2}, 0)$, at $\alpha=\alpha_{\mathrm{PS}}^{*}+0.0026$, where $\alpha_{\mathrm{PS}}^{*}=0.0984$ is the critical value at which sound velocity vanishes in a particular direction (P-criterion). Hydrostatic tension and pure shear strain correspond to paths 1 and 2, respectively, in figure 2e.

Figures $3 \mathrm{a}$ and $4 \mathrm{a}$ show the initial configurations with a small random perturbation of the atomic positions. Due to the instability, atoms start to move and structural reconstruction takes place. Figures $3 \mathrm{~b}$ and $4 \mathrm{~b}$ present the initial stages of reconstruction. In figure $3 \mathrm{~b}$ a microscopic crack appears, while in figure $4 \mathrm{~b}$ a pair of dislocations is formed. Figures $3 \mathrm{c}$ and $4 \mathrm{c}$ show the final results of the postcritical transformation. When the transformations are complete the crystals appear to be thermalized. Recall that, in our simulations, the computational cell has fixed shape. If we use the Parinello-Rahman scheme with fixed stress and variable lattice parameters then the volume of the computational cell would increase infinitely because the crystal under these conditions can be in equilibrium with the external stresses only if the latter decrease.

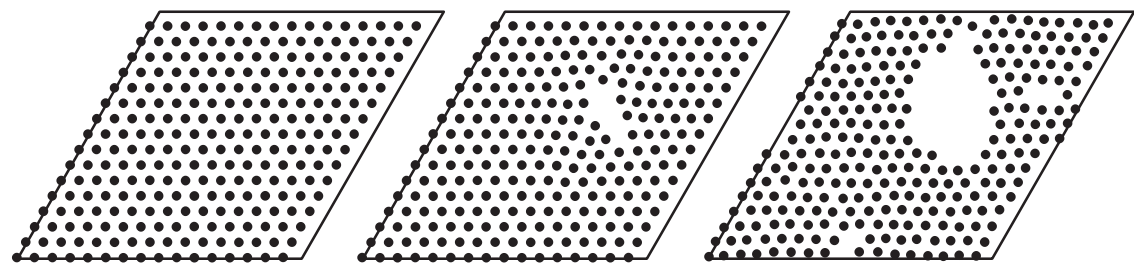

(a)

(b)

(c)

Figure 3. Post-critical behaviour of the hydrostatically stretched LJ lattice at $\alpha=\alpha_{\mathrm{HT}}^{*}-$ 0.0022 in the presence of small temperature.

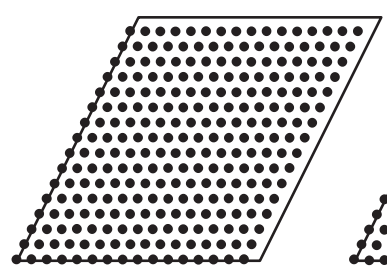

(a)

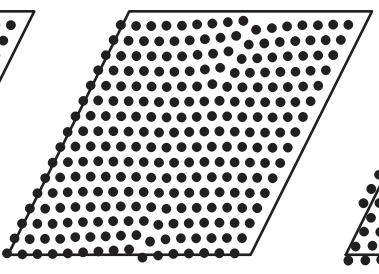

(b)

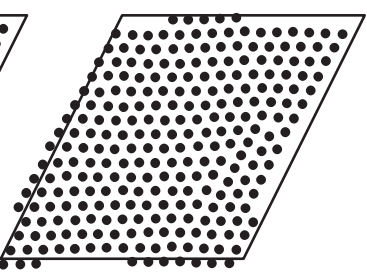

(c)

Figure 4. Same as figure 3, but for pure shear strain with $\alpha=\alpha_{\mathrm{PS}}^{*}+0.0026$. 
In figure 5 we present the time evolution of the kinetic and potential energies during the structural transformation shown in figure 3 . Here as the zero level for the potential energy we choose the cohesive energy $\mathrm{E}^{*}=0.186$ of the hydrostatically strained crystal with $\alpha=0.1575$. After some incubation period the structural transformation begins and the potential energy starts to decrease, while the kinetic energy (temperature) increases, with the total energy being conserved.

An interesting precursor to the collapse of the lattice under pure shear strain was observed. We found a bifurcation point at which the homogeneously strained structure becomes unstable and a modulation wave with small amplitude $\left(\sim 10^{-4} a_{0}\right)$ appears as shown in figure 6 . The structure is stable in a narrow interval of $\alpha$ and, with increasing $\alpha$, the modulation wave very soon becomes unstable and dislocation pairs are formed. This kind of structural transformation has been reported for a homogeneously strained fcc crystal [29]. The appearance of the small amplitude incommensurate modulation breaks the homogeneous distribution of energy in the crystal and facilitates the nucleation of dislocations $[22,29]$.

We have observed an influence of the size of the periodic computation cell, $N$, on the critical values. This issue can be very important for dislocation nucleation during nano-indentation because, in the presence of a strain gradient, the criterion of instability is fulfilled first in a local area and the nucleation of dislocations can be detained until this area reaches a critical size.

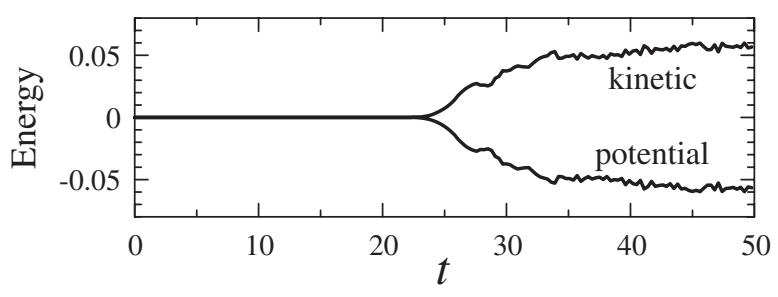

Figure 5. Time evolution of the potential and kinetic energies during the structural transformation of the hydrostatically stretched LJ crystal presented in figure 3.

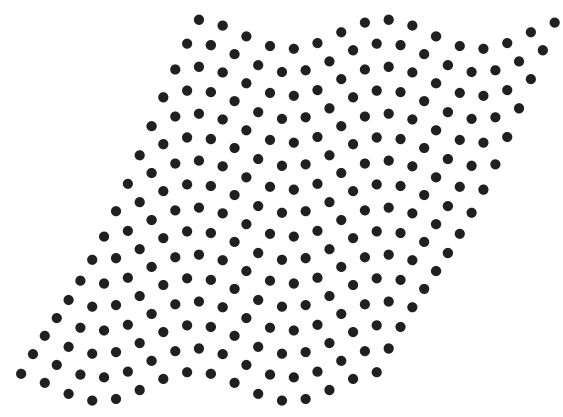

Figure 6. Stable modulated structure observed at $\alpha$ slightly lower than the dislocation nucleation point at pure shear. The amplitude of the modulation wave is very small $\left(\sim 10^{-4} a_{0}\right)$ and to make it visible we had to considerably enhance the atomic displacements with respect to the homogeneously strained lattice positions. The appearance of the small amplitude incommensurate modulation breaks the homogeneous distribution of energy in the crystal and facilitates the nucleation of dislocations. 
We found that the smaller $N$, the greater is the magnitude of $\alpha$ at which a structural transformation occurs in a marginally thermalized crystal (figure 7). Relative increases in $\alpha$ compared with the corresponding critical values found from the P-criterion for infinite crystals are shown by dots as a function of $N$ for hydrostatic tension (figure 7a) and pure shear strain (figure 7b). The critical values in figure $7 \mathrm{a}$ are a few percent smaller than $\alpha_{\mathrm{HT}}^{*}$. This is because, for hydrostatic tension, it is not the $\mathrm{P}$-criterion but the $\mathrm{B}$-criterion that gives a lower critical value. For the same reason, the size effect in this case is one order of magnitude smaller than in the case of pure shear strain, where, for example, for $N=4$ the increase in $\alpha$ is about $19 \%$ compared with $\alpha_{\text {PS. }}^{*}$.

The origin of the size effect is illustrated in figure 8 . Black areas in figure 8 show the points of the first Brillouin zone with imaginary frequencies for hydrostatic tension (figure 8a) at $\alpha=\alpha_{\mathrm{HT}}^{*}+0.0050$ and for pure shear strain (figure 8b) at $\alpha=\alpha_{\mathrm{PS}}^{*}+0.0050$. The first imaginary frequency points appear near the origin of
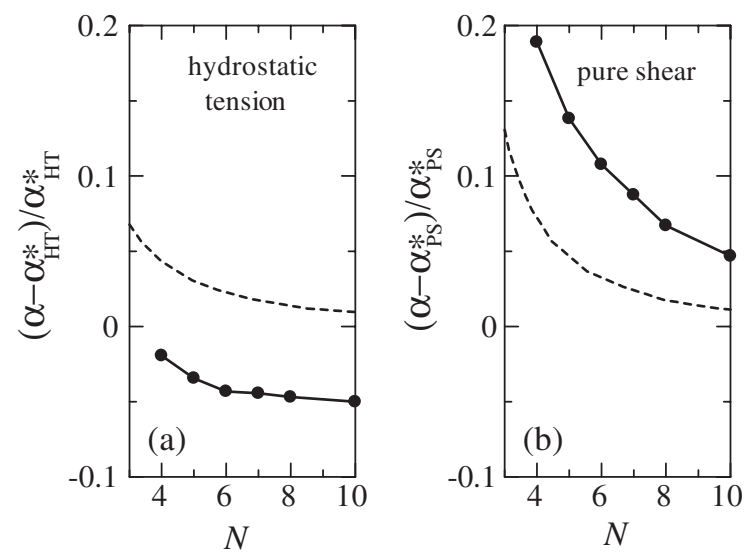

Figure 7. Effect of the size of the periodic computational cell, $N$, on the magnitude of $\alpha$ at which a structural transformation occurs in a marginally thermalized crystal. Dots show the relative change in $\alpha$ compared with the corresponding critical values found from the P-criterion for infinite crystals. Dashed lines show the shortest unstable waves (in units of $a$ ) for different $\alpha$, measured in units of the lattice spacing, $a$.

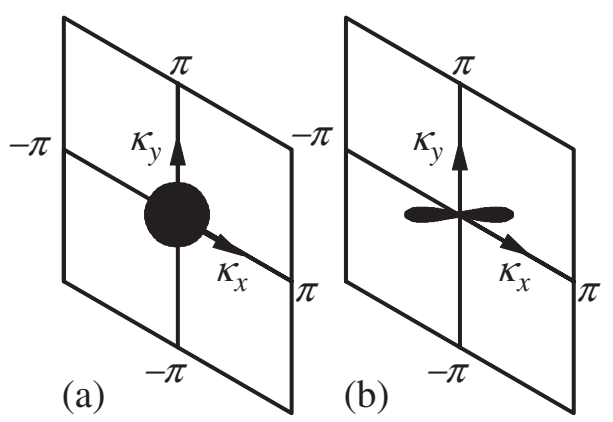

Figure 8. Black areas show the points of the first Brillouin zone with imaginary frequencies for (a) hydrostatic tension at $\alpha=\alpha_{\mathrm{HT}}^{*}+0.005$ and (b) for pure shear strain at $\alpha=\alpha_{\mathrm{PS}}^{*}+0.005$. 
the first Brillouin zone and then the black regions grow in size monotonically. For $\alpha$ only slightly greater than $\alpha^{*}$, the unstable waves have a large wavelength. If the wavelength of an unstable wave is greater than the size of the computation cell, the corresponding instability mode cannot be realized. Dashed lines in figure 7 show the shortest unstable waves for different $\alpha$, measured in units of the lattice spacing, $a$. The dashed curves correlate with the dots obtained numerically for the finite size computation cell.

\section{Indentation}

Here the results for the LJ potential will be reported since they differ only quantitatively from those for the BR potential.

In our indentation simulations we start from the defect-free system. System 1 (see section 2 for simulation details) deforms elastically until indentation depth 2.60, while system 2 deforms elastically until indentation depth 3.17. Pre-critical stable atomic structures for systems 1 and 2 are shown in figures 9 and 11, respectively.

We make an attempt to predict the location of the dislocation nucleation point in the crystal by applying the soft mode instability criterion (P-criterion). To do this, we define the local strain for each primitive cell of the indented crystal and calculate the dispersion curves for a crystal homogeneously strained with this strain. Open circles in figures 9 and 11 show the primitive cells for which the calculated spectrum contained imaginary frequencies. This criterion can be applied to predict the dislocation nucleation in the bulk of a defect-free crystal where the strain gradients are relatively small. For the lattice instabilities near the surface or other heterogeneities a more general approach should be used (see e.g. [30-32]). The actual dislocation nucleation and subsequent gliding are in very good agreement with the instability patterns. In system 1 , the pair of dislocations 1 and 2 and then the pair 3 and 4 (see figure 10) were nucleated in the middle of the unstable region of figure 9 . The dislocations glide along the closed-packed directions tilted by $\pi / 3$ and $2 \pi / 3$ with respect to the $x$ axis. The position of the dislocation nucleation point in this case is also in agreement with the experimental observation for bubble raft indentation [33]

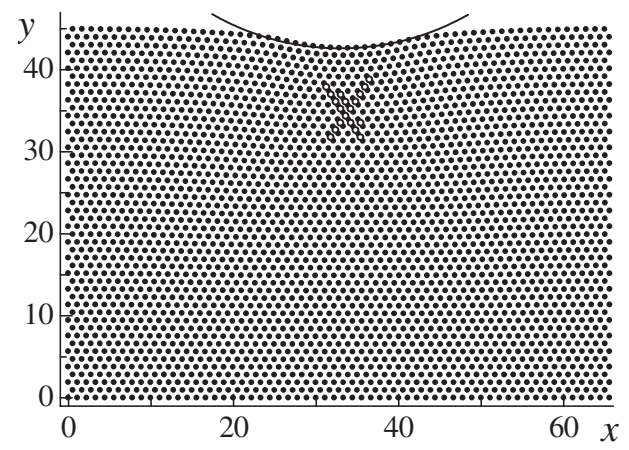

Figure 9. Indentation of system 1 (see text). Dots show the atoms and circles show the primitive cells where the soft mode criterion of instability is already satisfied. The indentation depth is 2.60 . 
and also with the prediction based on the two-dimensional Hertzian indentation theory (see e.g. [33]).

Dislocation nucleation and subsequent gliding observed for system 2 (figure 12) are also in very good agreement with the instability pattern of figure 11 . Note that here dislocations glide along the $y$ direction.

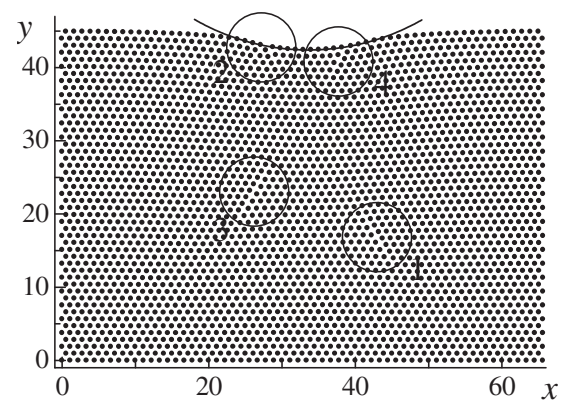

Figure 10. Stable configuration of system 1 at an indentation depth of 2.61. Four dislocations were nucleated, first the pair 1 and 2 and then the pair 3 and 4 . The nucleation centre and the dislocation gliding directions are in agreement with the instability pattern of figure 9 .

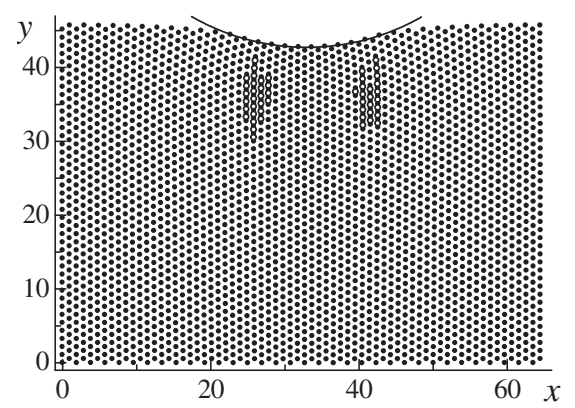

Figure 11. Same as figure 9, but for system 2 at an indentation depth of 3.17. In this case also, the dislocations are not nucleated until the volume of the unstable regions reaches a critical value.

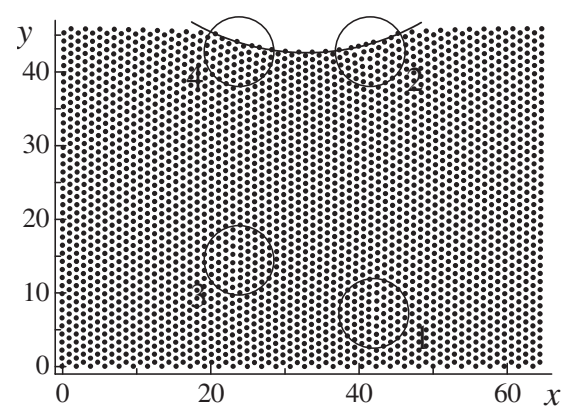

Figure 12. Same as figure 10, but for system 2 at an indentation depth of 3.19. Note that, in contrast to figure 10, dislocations glide in the vertical direction. Here also, the dislocation nucleation centres and the gliding directions are accurately predicted by the soft mode criterion (see figure 11). 


\section{Conclusions}

For a 2D crystal with two different interatomic potentials we have calculated the instability surfaces in the three-dimensional strain space and the crystal energy at the critical points. The latter result helped us to specify the loading conditions when lattice instability is most probable. The possibility of constructing the instability surfaces relies on the fact that the criterion of instability of an equilibrium state is path-independent. Some properties of instability surfaces are summarized in section 3.1. The soft mode instability criterion is applied successfully to predict the dislocation nucleation points and the dislocation gliding directions in the indentation of a $2 \mathrm{D}$ single crystal along the $\langle 112\rangle$ and $\langle 110\rangle$ directions.

We offer an approach to incorporate the effect of the interaction of the sample with the loading device into a criterion of lattice instability with respect to a homogeneous strain mode (section 3.2).

In nano-indentation experiments, mode softening occurs in a local area and it does not cause an immediate collapse of the lattice (nucleation of dislocations). Collapse occurs after the size of the unstable region reaches a threshold value. Analysing the distribution of the unstable modes over the first Brillouin zone we put forward an explanation for this behaviour. In the homogeneously strained 2D hexagonal crystal, mode softening occurs first infinitesimally close to the origin of the first Brillouin zone (vanishing of sound velocity). Thus, in the beginning, the crystal is unstable only with respect to very long waves. On a further increase in the homogeneous strain, the modes with shorter waves become unstable. Collapse of the crystal lattice in the presence of a strain gradient may occur when a mode with wavelength nearly equal to the size of the unstable region becomes soft. Note that the above is applicable to instability with respect to a phonon mode, but not with respect to a homogeneous strain mode.

We have systematically compared the results obtained with the use of the longrange Lennard-Jones potential equation (1) and the short-range potential equation (2) and found only quantitative differences. For example, both potentials applied to the instability of a homogeneously strained crystal (section 3) never gave an instability with respect to a short-wave phonon. It is easier to observe this type of instability for a crystal with complex structure but, in our case, the instability mechanism was always the vanishing of sound velocity. In the non-homogeneous set-up, i.e. in the indentation simulations, the difference was also only quantitative. In our recent studies $[34,35]$ of near-surface lattice instabilities we also observed that the structure and crystallographic orientation of the surface play a more important role in controlling the instability mechanisms than the actual law of interatomic interactions.

The size effect, i.e. the effect of the size of the periodic computational cell on the value of the external loading parameters when a collapse of the crystal lattice occurs, has been discussed. The effect is important only for instability with respect to a phonon mode. In this case we also observed an interesting precursor of lattice collapse, first reported by Dmitriev et al. [29] for a homogeneously strained fcc crystal. In a computational cell of finite size, a stable modulated structure is observed with a loading parameter slightly lower than the dislocation nucleation point and slightly higher than the mode softening point. The amplitude of the modulation wave increases with external load, but it does not reach a considerable value, and, in our numerical experiments, is of the order $10^{-4} a_{0}$. Soon after 
appearance the wave becomes unstable and the crystal lattice collapses. The appearance of the small amplitude incommensurate modulation prior to lattice collapse breaks the homogeneous distribution of energy in the crystal and facilitates the nucleation of dislocations.

We have demonstrated the reliability of the microscopic soft mode criterion (P-criterion) in predicting the dislocation nucleation centres and the gliding directions during indentation. Our approach is to define the local strain for each primitive cell of the indented crystal and calculate the dispersion curves for an infinite crystal homogeneously strained with this strain. However, this criterion can be successfully applied to predict the dislocation nucleation only in the bulk of a defect-free crystal, for example in nano-indentation, where the strain gradients are relatively small and the homogeneous consideration can be applied with a high expectation. For lattice instabilities near the surface or other heterogeneities a more general approach should be used (see e.g. [30-32, 34, 35]).

\section{Acknowledgements}

We thank Krystyn J. Van Vliet and Sidney Yip for the inspiring discussions during the IUTAM Meeting in Osaka, July 2003. We also thank Takayuki Kitamura, Yoshitaka Umeno and Kisaragi Yashiro for very helpful discussions. J.L. acknowledges support from Honda R\&D Co., Ltd. and the Ohio State University Transportation Research Endowment Program.

\section{References}

[1] Yu.A. Izyumov and V.N. Syromyatnikov, Phase Transitions and Crystal Symmetry (Kluwer, Dordrecht, 1984).

[2] J.-C. Tolédano and P. Tolédano, The Landau Theory of Phase Transitions (World Scientific, Singapore, 1987).

[3] V.V. Bolotin, Nonconservative Problems of the Theory of Elastic Stability (Pergamon Press, Oxford, 1963).

[4] R. Hill, Math. Proc. Camb. Phil. Soc. 77225 (1975).

[5] R. Peierls, Proc. phys. Soc. Lond. 5234 (1940).

[6] F.R.N. Nabarro, Proc. phys. Soc. Lond. 59256 (1947).

[7] J. Frenkel, Z. Phys. 37572 (1926).

[8] A.P. Levitt (Editor), Whisker Technology (Wiley, New York, 1970).

[9] W.W. Gerberich, J.C. Nelson, E.T. Lilleodden, et al., Acta mater. 443585 (1996).

[10] S. Suresh, T.G. Nieh and B.W. Choi, Scripta mater. 41951 (1999).

[11] A. Gouldstone, H.J. Koh, K.Y. Zeng, et al., Acta mater. 482277 (2000).

[12] J. Li, K.J. Van Vliet, T. Zhu, et al., Nature 418307 (2002).

[13] K.J. Van Vliet, J. Li, T. Zhu, et al., Phys. Rev. B 67104105 (2003).

[14] M.A. Biot, Mechanics of Incremental Deformation (Wiley, New York, 1965).

[15] P.W. Anderson, in Proceedings of the Conference on the Physics of Dielectrics, edited by G.I. Skanavi (Academy of Science, Moscow, 1958), p. 290.

[16] W. Cochran, Adv. Phys. 9387 (1960).

[17] Y. Yamada, Dynamical Properties of Solids, vol. 5, edited by G.K. Horton and A.A. Maradudin (North-Holland, Amsterdam, 1984), p. 329.

[18] R. Blinc and A.P. Levanyuk (Editors), Incommensurate Phases in Dielectrics, vols 1 and 2 (North-Holland, Amsterdam, 1986).

[19] Y. Shibutani, in Advances in Materials Research, edited by H. Kitagawa, T. Aihara and Y. Kawazoe (Springer, Berlin, 1998), p. 100.

[20] J. Wang, J. Li, S. Yip, et al., Phys. Rev. B 5212627 (1995). 
[21] R. Hill and F. Milstein, Phys. Rev. B 153087 (1977).

[22] J. Li, A.H.W. Ngan and P. Gumbsch, Acta mater. 515711 (2003).

[23] A.A. Vasiliev, S.V. Dmitriev, Y. Ishibashi, et al., Phys. Rev. B 65094101 (2002).

[24] S.V. Dmitriev, D.A. Semagin, T. Shigenari, et al., Phys. Rev. B 68052101 (2003).

[25] S.V. Dmitriev, A.A. Vasiliev and N. Yoshikawa, Recent Res. Devel. Phys. 4267 (2003).

[26] D.M. Clatterbuck, C.R. Krenn, M.L. Cohen, et al., Phys. Rev. Lett. 91135501 (2003).

[27] T. Janssen and J.A. Tjon, Phys. Rev. B 253767 (1982).

[28] J. Li, T. Zhu, S. Yip, et al., Mater. Sci. Engng. A 36525 (2004).

[29] S.V. Dmitriev, A.A. Ovcharov, M.D. Starostenkov, et al., Phys. Solid St. 38996 (1996).

[30] T. Kitamura, Y. Umeno and N. Tsuji, Comp. Mater. Sci. 29499 (2004).

[31] T. Kitamura, Y. Umeno and R. Fushino, Mater. Sci. Engng. A 379229 (2004).

[32] K. Yashiro and Y. Tomita, J. Phys. IV France 115 (2001).

[33] A. Gouldstone, K.J. Van Vliet and S. Suresh, Nature 11656 (2001).

[34] S.V. Dmitriev, J. Li, N. Yoshikawa, et al., in Defects and Diffusion in Metals, Annual Retrospective 2004, edited by D.J. Fisher, p. 49.

[35] S.V. Dmitriev, T. Kitamura, J. Li, et al., Acta Mater. 531215 (2005). 